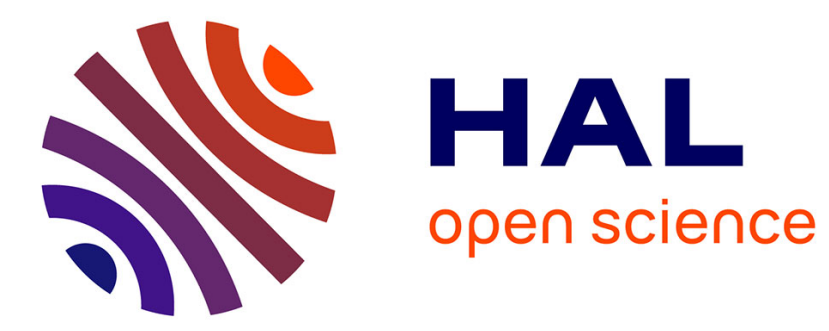

\title{
Du nouveau sur la bibliothèque de Benoît Lecourt
}

Hélène Lannier, William Kemp

\section{To cite this version:}

Hélène Lannier, William Kemp. Du nouveau sur la bibliothèque de Benoît Lecourt. Réforme, Humanisme, Renaissance, 2014. hal-01890436

\section{HAL Id: hal-01890436 https://hal.science/hal-01890436}

Submitted on 8 Oct 2018

HAL is a multi-disciplinary open access archive for the deposit and dissemination of scientific research documents, whether they are published or not. The documents may come from teaching and research institutions in France or abroad, or from public or private research centers.
L'archive ouverte pluridisciplinaire $\mathbf{H A L}$, est destinée au dépôt et à la diffusion de documents scientifiques de niveau recherche, publiés ou non, émanant des établissements d'enseignement et de recherche français ou étrangers, des laboratoires publics ou privés. 


\section{Du nouveau sur la bibliothèque de Benoît Lecourt ${ }^{1}$}

Hélène LANNIER, Université Lumière Lyon 2

William KEMP, Université McGill

La bibliothèque de Benoît Lecourt, un érudit qui prit part à la vie littéraire lyonnaise dans la première moitié $\mathrm{du} \mathrm{XvI}^{\mathrm{e}}$ siècle, est connue depuis longtemps mais n'a jusqu'à présent fait l'objet que d'études partielles². Des reliures à ses armes sont signalées dans plusieurs bibliothèques. La British Library conserve notamment depuis 1838 un volume factice qui rassemble trente-trois opuscules d'origines italienne et française datant environ de 1485 à $1539^{3}$. D'après le Catalogue of books printed in the Xvth century now in the British Museum (BMC), les armes présentes sur la

1. L'orthographe française du nom de famille de Benoît Lecourt est encore aujourd'hui fluctuante. Dans les commentaires en français du XviII ${ }^{\mathrm{e}}$ au XxI ${ }^{\mathrm{e}}$ siècle, on trouve aussi bien Benoît Court que Benoît Le Court, Benoît Lecourt, Benoît de Court ou encore Benoît de Curtil. Pour la plupart, les documents d'époque qui le concernent sont en latin et indiquent: Benedictus Curtius; ils ne permettent donc pas de statuer sur la particule. Nous avons cependant choisi de privilégier "Lecourt » car c'est l'orthographe que le seul document en français qui concerne directement notre homme, trouvé lors de nos recherches en archives, utilise. De plus, c'est celle employée par son frère, Léonard, pour signer les actes notariés.

2. Hélène Lannier prépare actuellement une thèse de doctorat sur Benoît Lecourt et est soutenue par une allocation doctorale de la Région Rhône-Alpes.

3. Nous tenons à exprimer notre gratitude au Dr Martin Davies, former Curator of Incunabula at the British Museum, à l'origine du projet Incunabula Short Title Catalogue (ISTC), qui a eu l'extrême gentillesse de vérifier les nombreuses attributions et datations des pièces incunables, en l'occurrence, les $n^{\circ} 1$ à 20 et 22 à 25 du recueil factice. Nos remerciements vont également à M. John Goldfinch, responsable de l'Incunabula and Early Western Printed Collection du département des British and Early Printed Collections de la British Library dont l'expertise nous a aidés à réaliser l'étude matérielle de ce recueil. Ce travail a également bénéficié des lectures attentives de Michèle Clément, Florence Desnoyers et Raphaële Mouren, nous les en remercions. 
reliure $^{4}$ de ce recueil sont celles de Benoît Lecourt ${ }^{5}$ - Benedictus Curtius, en latin. Cette thèse est confirmée par l'Armorial des bibliophiles de Lyonnais, Forez, Beaujolais et Dombes qui répertorie un fer (le $\left.\mathrm{n}^{\circ} 3\right)$ présentant un blason ainsi décrit: « d'azur à trois molettes d'or, 2 et $1^{6}$ ", identique à celui apposé sur la reliure du volume de la British Library. En outre, dans son article « À propos de Benoît Le Court » paru en 1999, Guy Parguez identifie plusieurs reliures de la Bibliothèque municipale de Lyon attribuées à Benoît Lecourt et portant ce même fer ${ }^{7}$. En plus de ces données, le recueil comporte en lui-même des éléments qui confirment sa provenance. La signature de Benoît Lecourt en latin "Benedictus Curtius Lugdunensis » se trouve en effet au bas de la page de titre du vingt-sixième opuscule du volume ${ }^{8}$, Coram Iulio secundo appologia, de Guillaume Briçonnet, paru à Lyon chez Vincent de Portonariis en $1507^{9}$. C'est peut-être également Benoît Lecourt qui a inscrit en espagnol, de part et d'autre des armes de France imprimées sur cette même page de titre ${ }^{10}$, la phrase suivante: "Diva Francia sobre mar y tierra ». Il a de nouveau signé ce texte au dos du dernier feuillet (e4v) dans le style de Grolier ${ }^{11}$ mais en substituant sa

4. Pour une vue de la reliure se référer à la figure 1 .

5. Catalogue of books printed in the Xvth century now in the British Museum [BMC]. Part IV. Italy: Subiaco and Rome, London, British Museum, 1916, p. 95.

6. L'expression « 2 et 1 » précise la disposition des molettes sur le blason. Ici, l'une des molettes est placée en dessous des deux autres, voir Julien Baudrier, Léon Galle, William Poidebard, Armorial des bibliophiles de Lyonnais, Forez, Beaujolais et Dombes, Lyon, Société des bibliophiles lyonnais, 1907 , p. 347.

7. Guy Parguez, "À propos de Benoît Le Court », dans Actes des journées d'études 1998, XV, SaintSymphorien-sur-Coise et sa région, Lyon, Archives départementales du Rhône, 1999, p. 21-31.

8. Pour une vue de cette page se reporter à la figure 2 .

9. Coram Iulio secundo: Maximo pontifice: Sacroque Cardineo collegio: Pro christianissimo francorum rege Ludovico. XII. aduersus impudentem \& parum consultum calumniatorem appologia: Per Reuerendissimum. d. d. Gulielmum Briconnentum [...], Lyon, Vincent de Portonariis, 1507. Cette édition est décrite par Henri Baudrier dans la Bibliographie Lyonnaise. Recherches sur les imprimeurs, libraires, relieurs et fondeurs de lettres de Lyon au xvI siècle, tome V, Paris, F. de Nobele, 1964, p. 404 et par Sybille von Gültlingen dans sa Bibliographie des livres imprimés à Lyon au seizième siècle, Baden-Baden et Bouxwiller, tome II, Éditions V. Koerner, 1993 (Bibiotheca bibliographica Aureliana, 141), p. 161. Voir aussi l'annexe 1 du présent article. Il s'agit de l'une des premières éditions datées de V. de Portonariis.

10. D’après Henri Baudrier, op. cit., p. 404 : « Le titre, blanc au verso, porte, au recto, les armes de France, portées par un porc-épic et entourées du collier de Saint-Michel et deux tiges de lis. »

11. À ce sujet, voir l'article de Geoffrey Dudley Hobson, "Et amicorum », The library, IV, n 2, septembre 1949, p. 87-99. 
ville natale, St-Symphorien à Lyon: "Benedicti Curtii Symphoriani et amicorum ». Ajoutons que le frère de Benoît Lecourt, Léonard, a également laissé sa signature à la fin de ce texte. On trouve enfin l'inscription «Benedictus Curtius Symphorianus » au dos du dernier feuillet de la vingt-huitième pièce (f. a4v), l'Oratio apud Leonem decimum de Stephan Possedarski publiée à Rome chez Stefano Guillery vers $1517^{12}$. Autre élément remarquable, presque toutes les pièces sont numérotées au moyen d'une lettre simple de $a$ à $z$, puis d'une lettre double de $a a$ à $h h^{13}$. Cette numérotation, quasiment continue, a été réalisée par une seule main.

Le recueil aujourd'hui conservé à la British Library a donc eu un jour sa place parmi les livres de la bibliothèque de Benoît Lecourt et étant donné que le dernier texte du volume, qui est aussi le plus récent, les Binae orationes de Bartholomaeus Frexius ${ }^{14}$, est paru chez Sébastien Gryphe en 1539, nous pouvons affirmer que cet ensemble de textes a été relié pour Benoît Lecourt après cette date.

Le contenu intellectuel d'un livre est évidemment révélateur des centres d'intérêt de son possesseur. Mais encore, un examen approfondi des données matérielles d'un volume, surtout dans le cas des constitutions complexes des recueils factices, peut permettre d'appréhender les pratiques de lecture et les pratiques bibliophiliques des gens du temps. Ces données prennent encore une autre dimension lorsque la provenance du livre étudié est connue. Aussi l'analyse autant intellectuelle que matérielle d'un recueil factice ayant appartenu à Benoît Lecourt permet-elle de mettre en perspective le contenu des éditions qui le composent et les étapes de leur assemblage avec les données biographiques et les intérêts intellectuels de leur possesseur.

12. Attribution aux presses de Stefano Guillery et datation approximative d'après le Short-title catalogue of books printed in Italy and of Italian books printed in other countries from 1465 to 1600 now in the British Museum, London, British Museum, 1958, p. 537.

13. Voir annexe 1.

14. Édition décrite par Henri Baudrier, op. cit., tome VIII, Paris, F. de Nobele, 1964, p. 123. Voir aussi l'annexe 1 de cet article. 


\section{Benoît Lecourt, un bibliophile au $\mathrm{XVI}^{\mathrm{e}}$ siècle}

Originaire de Saint-Symphorien-le-Château - aujourd'hui SaintSymphorien-sur-Coise -, une commune située à une quarantaine de kilomètres de Lyon, Benoît Lecourt naît à la fin du Xv siècle ${ }^{15}$. Issu d'une famille de notables, il entreprend des études de droit et obtient le grade de docteur in utroque iure. C'est à ce titre, mais aussi en tant que curé de Coise, qu'il est nommé chevalier de l'Église de Lyon en 1540, une charge qu'il remplit jusqu'à sa mort, en 1559. Au nombre de sept, les chevaliers constituaient le service juridique des chanoines-comtes de Lyon, rattachés au chapitre de la primatiale Saint-Jean ${ }^{16}$.

Benoît Lecourt est également connu pour être l'auteur de trois ouvrages éclectiques publiés à Lyon. C'est en tant que jurisconsulte et docteur in utroque iure qu'il écrit un manuel expliquant les termes de droit romain et canon, l'Enchiridion iuris utriusque terminorum, publié par les frères Frellon en $1543^{17}$. Il est aussi l'auteur d'un ouvrage sur les jardins, l'Hortorum libri triginta, publié chez Jean de Tournes en $1560^{18}$. Mais il est surtout connu pour avoir proposé un commentaire juridique en latin au texte français des Arrêts d'amours de Martial d'Auvergne, renommés Arresta amorum pour l'occasion. La première édition paraît à Lyon, chez Sébastien Gryphe, en 1533. Puis le commentaire est réédité à huit reprises jusqu'au XviII ${ }^{\mathrm{e}}$ siècle, accompagnant toujours le texte français de Martial d'Auvergne ${ }^{19}$.

15. Guy Parguez, op. cit., p. 21.

16. Au sujet des chevaliers de l'Église de Lyon voir Adolphe Vachet, Les Anciens Chanoines-comtes de Lyon, Lyon, Imprimerie Emmanuel Vitté, 1897 et Jean Beyssac, Les Chevaliers de l'Église de Lyon, Lyon, Imprimerie des missions africaines, 1925.

17. Enchiridion iuris utriusque terminorum, Benedicto Curtio Symphoriano, equite Ecclesiae Lugdunensis, auctore [...], Lyon, Jean et François Frellon, 1543. Édition décrite dans Henri Baudrier, op. cit., tome V, Paris, F. de Nobele, 1964, p. 189-190.

18. Hortorum libri triginta. Autore Benedicto Curtio Symphoriano, equite in Ecclesia Lugdunensi. In quibus continentur arborum historia [...], Lyon, Jean de Tournes, 1560. Euvre décrite par Alfred Cartier dans sa Bibliographie des éditions des de Tournes, imprimeurs lyonnais. Mise en ordre avec une Introduction et des Appendices par Marius Audin et une Notice biographique par E. Vial, Lyon, tome II, Paris, Éditions des Bibliothèques nationales de France, 1937, p. 503.

19. Arresta amorum. Cum erudita Benedicti Curtii Symphoriani explanatione, Lyon, Sébastien Gryphe, 1533. Voir Henri Baudrier, op. cit., tome VIII, Paris, F. de Nobele, 1964, p. 68 et Sybille von Gültlingen, op. cit., tome V, 1997 (Bibiotheca bibliographica Aureliana, 160), p. 48. 
D'après les épigrammes de Claude Rousselet ${ }^{20}$ et de Gilbert Ducher ${ }^{21}$, nous pouvons, à la suite de V.-L. Saulnier, situer Benoît Lecourt parmi les lettrés lyonnais des années 1530-154022. Ce sont peut-être ces témoignages qui ont incité les biographes du XviII ${ }^{\mathrm{e}}$ siècle à en faire un membre de la mythique Académie de Fourvière, une assemblée savante qui se serait réunie chez l'antiquaire lyonnais Guillaume Du Choul, dans sa maison de la montée du Gourguillon ${ }^{23}$. Si l'existence de cette Académie est contestée, on est cependant certain que Benoît Lecourt a entretenu des liens avec certains des membres du sodalitium lyonnais des années 1530-1560.

De plus, en homme cultivé, le juriste a constitué une collection de livres dont nous pouvons aujourd'hui retracer en partie l'histoire. Car, suivant l'habitude des collectionneurs, il faisait couvrir ses livres d'une reliure portant ses armes. Le recueil de la British Library en fournit un bel exemple. Il présente une reliure aux armes en veau brun avec un dos à cinq nerfs. Sur chacun de ses plats (de $183 \mathrm{~mm}$ de hauteur sur $138 \mathrm{~mm}$ de largeur) se dessinent un encadrement de filets estampés à froid avec des fleurons estampés à chaud aux quatre coins. Enfin, on y trouve les restes de fermoirs cloutés ${ }^{24}$.

C'est au château de Pluvy, propriété de la famille Lecourt depuis le XIV ${ }^{e}$ siècle, que les livres de Benoît Lecourt auraient été conservés de son vivant. D'après les auteurs de l'Armorial des bibliophiles, la bibliothèque aurait été divisée en deux parts vers 1588, bien après la mort du juriste $^{25}$. Une partie des livres aurait alors quitté le château, à ce moment

20. Claudii Rosseletti, iureconsulti, Patritiique Lugdunensis Epigrammata, Lyon, Sébastien Gryphe, 1537, p. 46. Voir Henri Baudrier, op. cit., tome VIII, Paris, F. de Nobele, 1964, p. 102-103 et Sybille von Gültlingen, op. cit., tome V, 1997 (Bibiotheca bibliographica Aureliana, 160), p. 78.

21. Gilberti Ducherii Vultonis aquapersani epigrammaton libri duo, Lyon, Sébastien Gryphe, 1538, p. 143. Voir Henri Baudrier, op. cit., p. 113-114 et Sybille von Gültlingen, op. cit., p. 82.

22. V.-L. Saulnier, Maurice Scève (ca. 1500-1560), Paris, Librairie C. Klincksieck, 1948, tome premier, p. 90 et suiv.

23. Jacques Pernetti, Recherches pour servir à l'histoire de Lyon, ou les Lyonnois dignes de mémoire. Tome Premier, À Lyon, chez les Frères Duplain, Libraires, grande rue Mercière, 1767, avec Privilège du Roi, p. 328-329.

24. Voir la figure 1. À l'intérieur du volume, les blocs de textes mesurent environ 176 sur $128 \mathrm{~mm}$.

25. Jean Baudrier, Léon Galle, William Poidebard, op. cit., p. 348. 
détenu par Angelin Lecourt, neveu de Benoît et fils de Léonard. On a prétendu en revanche que la seconde partie de la bibliothèque y aurait été conservée, peut-être jusque dans les années 1970, au moment du rachat du château par la ville de Saint-Symphorien-sur-Coise aux descendants de la famille Lecourt ${ }^{26}$. Cependant, aucun document ne nous permet à ce jour de prouver que cette partie de la bibliothèque ait suivi le sort du château jusqu'au $\mathrm{xx}^{\mathrm{e}}$ siècle. À ce jour, nous avons recensé un peu moins de cinquante volumes reliés aux armes de Benoît Lecourt, et/ ou portant sa signature et/ou des annotations de sa main. Ces ouvrages sont dispersés dans les bibliothèques publiques en France et à l'étranger. Le repérage est beaucoup plus difficile à effectuer mais il est certain que des reliures de Benoît Lecourt sont également conservées dans des collections privées, ce qui laisse supposer un volume total de la bibliothèque plus important. La plus grande concentration de reliures de Benoît Lecourt - soit un peu plus d'une vingtaine de volumes - est conservée à la Bibliothèque municipale de Lyon ${ }^{27}$. En ce qui concerne le recueil qui nous intéresse ici, les raisons qui l'ont amené à voyager des Monts du lyonnais jusqu'à Londres sont encore inconnues.

\section{Un recueil d'Orationes officielles}

Examinons maintenant le contenu du volume factice de Benoît Lecourt. D’une épaisseur moyenne, il ne contient pourtant pas moins de trentetrois éditions distinctes comptant pour la plupart entre un et dix feuillets in-quarto. L'annexe 1 décrit succinctement ces éditions en donnant leur cotation d'origine en lettres et une numérotation correspondant à leur place dans le recueil. Chaque pièce contient un discours solennel ayant été prononcé à Rome par un orateur sacré ou un ambassadeur officiel

26. Ibid., p. 348.

27. Guy Parguez, op. cit., p. 23. Voir aussi le dossier thématique consacré à Benoît Lecourt dans la bibliothèque numérique de la Bibliothèque municipale de Lyon: Numelyo, Un bibliophile d'exception: Benoît Le Court (14..-1559) [en ligne], Lyon, Bibliothèque municipale de Lyon, [consulté le 04/12/2013], disponible à l'adresse: <http://numelyo.bm-lyon.fr/f_view/BML:BML_00GOO01 001THM0001lecour>. 
devant les papes et le Collège des cardinaux ${ }^{28}$. Étape incontournable de certaines cérémonies de la cour de Rome, ces orationes, destinées à être ensuite publiées, étaient prononcées en des occasions que l'on peut réunir en grandes catégories dont le recueil étudié donne des exemples ${ }^{29}$. On y trouve en effet des allocutions prononcées lors des célébrations de fêtes religieuses. C'est par exemple à l'occasion du jour de la Toussaint, le $1^{\mathrm{er}}$ novembre 1483, que Thomas de Capitaneis a présenté son discours devant Sixte IV ( $\left.n^{\circ} 3\right)$.

Les orationes peuvent aussi prendre la forme d'oraisons funèbres déclamées à l'occasion des funérailles de membres du haut clergé ou de princes laïques ${ }^{30}$, comme le discours que Leonello Chieregato a prononcé le 28 juillet 1492 lors des funérailles du pape Innocent VIII ( $\left.\mathrm{n}^{\circ} 11\right)$.

Le volume factice de Benoît Lecourt donne également plusieurs exemples d'orationes liées à la politique menée par les papes. Le discours $n^{\circ} 20$, par exemple, a été récité par Leonello Chieregato, prélat au service d'Innocent VIII puis d'Alexandre VI, à l'occasion du traité passé entre ce dernier, les ducs de Venise et de Milan et les rois de Rome et d'Espagne pour contrecarrer l'expédition militaire que Charles VIII menait sur Naples. La version imprimée du discours est accompagnée de la Bulle Quoniam pro communi, datée du 8 avril 1495, qui constitue le texte officiel de l'accord passé entre le pape et les Princes chrétiens. En tant qu'ambassadeur pour le roi de France, Guillaume Briçonnet a prononcé quant à lui un discours devant le pape Jules II ( $\left.{ }^{\circ}{ }^{26}\right)$. En juin 1507, Louis XII s'était réconcilié avec Ferdinand II d'Aragon lors d'une entrevue à Savone. Ce rapprochement et des projets apparemment

28. Pour une étude historique du cérémonial à la cour de Rome: Catherine Brice et Maria Antonietta Visceglia (éd.), Cérémonial et Rituel à Rome ( $X V I^{e}-X I X^{e}$ siècle), Rome, École française de Rome, 1997 (Collection de l'École française de Rome, 231), part. M. A. Visceglia, « Il cerimoniale come linguaggio politico. Su alcuni conflitti di precedenza alla corte de Roma tra Cinquecento e Seicento » p. 117-176.

29. Voir Denise Hillard, « Discours et sermons imprimés à Rome à la fin du $\mathrm{xv}^{\mathrm{e}}$ siècle à travers deux recueils de la Bibliothèque nationale de France », Bulletin du bibliophile, 2001, $\mathrm{n}^{\circ}$ 1, p. 38-40 et Holger Nickel, « Orations crossing the Alps ", dans Kristian Jensen (dir.), Incunabula and their readers: printing, selling and using books in the fifteenth century, Londres, British Library, 2003, p. 153.

30. John M. McManamon, Funeral oratory and the cultural ideals of Italian humanism, Chapell Hill et Londres, University of North Carolina Press, 1989, XIV-343 p. 
en discussion entre les deux monarques inquiétaient le pape, d'autant que de son côté l'empereur Maximilien le mettait en garde contre les entreprises de Louis XII, l'accusant de projeter de mauvais desseins contre le Saint Siège. Pour se disculper de ces accusations calomnieuses, Louis XII envoya Comes Carpi et Guillaume Briçonnet en ambassade à Rome. Ce dernier, évêque de Lodève en Languedoc, prononça alors, aux alentours du 25 mars 1507, un discours en faveur du roi de France devant le pape et le Collège des cardinaux. Plusieurs éditions du texte latin ont été publiées dès 1507 à Lyon, puis à Rouen et Paris. C'est l'édition de Lyon, la plus ancienne, qui se trouve dans le recueil qui nous intéresse ${ }^{31}$. Enfin, le discours $n^{\circ} 2$ présente un autre exemple d'allocution liée à la politique menée par les papes. Il s'agit d'une harangue contre les Turcs prononcée par Bernardo Giustiniano devant Sixte IV. Ce texte renvoie au problème posé par l'Islam au monde chrétien qui est, à cette époque, le principal sujet d'inquiétude pour les papes, d'où sa récurrence dans les discours ${ }^{32}$.

Enfin, la majorité des textes réunis dans le recueil qui nous intéresse sont des discours d'obédience adressés aux papes à l'occasion de divers événements officiels ${ }^{33}$. De plus, parmi ceux-ci, on remarque que les textes consacrés à Alexandre VI (treize discours) et à Innocent VIII (neuf discours) ${ }^{34}$ constituent une majorité écrasante. Celle-ci est tout à fait représentative de la production totale des discours puisque cette dernière compte non seulement une majorité de textes d'obédience mais aussi une forte proportion de pièces consacrées à ces deux papes ${ }^{35}$.

L'élection d'un souverain pontife était l'événement qui entraînait particulièrement abondance de discours. Comme le voulait l'usage, les

31. Michel Veissière, L'Évêque Guillaume Briçonnet (1470-1534): contribution à la connaissance de la réforme catholique à la veille du Concile de Trente, Provins, Société d'histoire et d'archéologie, 1986, p. 63-64.

32. Denise Hillard, op. cit., p. 40.

33. Les discours n ${ }^{\circ} 1,4$ à 10,13 à 15,17 à 19,21 à 25,27 et 29 à 32 .

34. Cf. annexe 2.

35. P. Casciano, G. Castoldi, M. P. Critelli; G. Curcio, P. Farenga Capriolo et A. Modigliani, « Materiali e ipotesi per la stampa a Roma ", Scrittura, biblioteche e stampa a Roma nel Quattrocento, Città del Vaticano, Biblioteca Apostolica Vaticana, 1980, tome I, p. 227. 
états catholiques envoyaient une ambassade pour prêter obédience au nouveau pape ${ }^{36}$. Or, le cérémonial comprenait la lecture d'un discours par l'un des membres de l'ambassade ${ }^{37}$. Ainsi plusieurs des orationes du recueil de Benoît Lecourt ont été prononcées au nom des principautés italiennes ou des souverains européens pour prêter obédience devant Alexandre VI après son élection en 1492. Placée à la suite de l'oraison funèbre destinée à son prédécesseur Innocent VIII, cette série d'allocutions prend tout son sens. Ainsi des discours ont-ils été prononcés par des ambassadeurs pour Florence ( $\left.\mathrm{n}^{\circ} 14\right)$, Gênes ( $\left.\mathrm{n}^{\circ} 18\right)$, Bertinoro $\left(n^{\circ} 19\right)$, Mantoue ( $\left.n^{\circ} 21\right)$, Venise ( $\left.n^{\circ} 22\right)$, Lucques ( $\left.n^{\circ} 23\right)$, Sienne $\left(n^{\circ} 24\right)$ et pour le duc de Milan ( $\left.n^{\circ} 25\right)$. On trouve aussi le discours de Nicolas d'Este au nom d'Hercule d'Este, duc de Ferrare et de Modène ( $\left.\mathrm{n}^{\circ} 15\right)$. À ceux-ci s'ajoutent le discours n ${ }^{\circ} 17$ récité par Pietro Cara pour le duc de Savoie et le n 12 par Bernardino de Carvajal pour Ferdinand d'Aragon et Isabelle de Castille, les rois d'Espagne. Citons un autre exemple de discours d'obédience, fort intéressant notamment du fait de sa rareté: d'après Richard Cooper ${ }^{38}$, l'exemplaire de la British Library est en effet l'unique témoin du discours prononcé par le savoyard Antoine Du Saix, commandeur de Saint-Antoine de Viennois, devant le pape Clément VII $\left(n^{\circ} 32\right)$. L'allocution a eu lieu à Marseille, lors du séjour que le souverain pontife y fit pour assister au mariage célébré le 28 octobre 1533 entre sa nièce, Catherine de Médicis, et le duc Henri d'Orléans. Antoine Du Saix a prononcé ce texte pour le compte de son ordre, les antonins, au nom de l'abbé de Saint-Antoine-de-Viennois, Antoine de Langeac, et de son frère Jean, le nouvel évêque de Limoges et vicaire général de l'ordre.

36. Sur les obligations d'obédience à l'élection d'un nouveau pape et sur les étapes du cérémonial voir René A. de Maulde-La-Clavière, La Diplomatie au temps de Machiavel, t. I, Paris, E. Leroux, 1892, p. 110-118.

37. L'ambassade florentine envoyée à Rome à l'avènement du pape Jules III ainsi que le discours prononcé par Piero Vettori sont par exemple l’objet d'une étude détaillée de Raphaële Mouren : «La rhétorique antique au service de la diplomatie moderne: Piero Vettori et l'ambassade florentine au pape Jules II (1550) ", Journal de la Renaissance, I, 2000, p. 121-154.

38. Richard Cooper, "Antoine du Saix, poète, diplomate et transfuge savoyard », dans Jean Balsamo (éd.), Mélanges de poétique et d'histoire littéraire $d u x v I^{e}$ siècle offerts à Louis Terreaux, Paris, Honoré Champion, 1994, p. 452. 


\section{De Rome à Lyon, acquisition et constitution du recueil}

Considérons à présent la nature même des volumes factices. Une collection peut contenir des textes de nature homogène ou hétéroclite, et le volume peut avoir été relié immédiatement, tardivement ou encore, il peut avoir connu plusieurs reliures.

On s'en rend compte, ce ne sont pas les auteurs des discours qui font l'unité du volume. Seul le nom de Leonello Chieregato ( $n^{\circ} 11$ et 20) y apparaît en effet deux fois. La plupart des auteurs sont italiens, mais on rencontre également des noms espagnols et polonais, et dans la seconde moitié du volume, constituée de textes du Xvi ${ }^{\mathrm{e}}$ siècle, des noms français, tels Guillaume Briçonnet et Antoine Du Saix. Les lieux d'édition ne contribuent pas non plus à créer un ensemble homogène. En effet, la majorité des discours qui composent le volume a été publiée à Rome par Stephan Planck (quinze discours sur trente-trois), Eucario Silber (six discours), Andreas Freitag et Stephan Guillery (un discours chacun). Cependant, certaines orationes ont été imprimées dans d'autres villes italiennes: Turin, Milan, Parme, Pavie. Puis, parmi les éditions datant du début du $\mathrm{XVI}^{\mathrm{e}}$ siècle, deux ont été produites à Bologne, une à Turin et enfin trois à Lyon.

Cependant, une homogénéité " générique » est assurée à l'ensemble par la nature des textes qui composent le volume. Bien plus, la cohérence du recueil est renforcée par le classement des discours suivant la succession chronologique des règnes des papes. Chaque titre comporte en effet le nom du pape devant lequel ou en l'honneur duquel l'oratio en question a été prononcée et de Calixte III à Paul III, presque tous s'y retrouvent, comme le met en évidence l'annexe 2. En plus du classement chronologique, la distribution des opuscules dans le recueil présente encore une autre particularité: selon les datations $\mathrm{BMC}^{39}$ complétées par celles de l'ISTC ${ }^{40}$, les vingt-cinq premières pièces, même celles

39. Catalogue of books printed in the Xvth century now in the British Museum [BMC], Part IV. Italy: Subiaco and Rome, London, British Museum, 1916, XVI-145 p.

40. Incunabula short title catalogue [en ligne], Londres, British Library, [consulté le 04/12/2013], disponible à l'adresse: <http://www.bl.uk/catalogues/istc/>. 
concernant les papes les plus reculés dans le temps, ont été imprimées entre 1481 et 1495 environ. Ce groupe de textes, dont aucun ne dépasse dix feuillets, représente $75 \%$ des textes du volume. Il semble donc que ces vingt-cinq discours constituent le noyau initial du recueil, d'autant qu'une période de douze années les sépare de la première des éditions du Xvi ${ }^{\mathrm{e}}$ siècle, l'Apologie de Briçonnet de 1507 (discours n ${ }^{\circ} 26$ ). On peut dès lors imaginer que cet ensemble a d'abord eu une vie autonome et que le recueil a été constitué en deux fois. Une première collecte de textes a vraisemblablement été réalisée en Italie à la fin $\mathrm{du} \mathrm{Xv}^{\mathrm{e}}$ siècle. Puis, les textes du XVI ${ }^{\mathrm{e}}$ siècle ont été ajoutés, marquant le recueil d'une influence française avec les noms des rois de France les plus récents : Louis XII et François ${ }^{\text {er }}$, et une contribution lyonnaise avec les éditions de Vincent de Portonariis et de Sébastien Gryphe.

L'influence française peut également être perçue en filigrane. Il est en effet intéressant de constater que la période de constitution de la première partie du recueil correspond aux dates de la première campagne d'Italie menée par Charles VIII entre septembre 1494 et juillet 1495 qui inclut un séjour d'environ un mois à Rome, du 31 décembre 1494 au 28 janvier 1495. Il est alors tentant d'imaginer qu'une série d'orationes concernant quelques papes de Calixte III à Alexandre VI ait été acquise et ramenée en France à ce moment. Il ne s'agit là que d'une hypothèse. Il semble cependant que la mise en commun de cette série se soit initialement faite à Rome, peut-être dans la boutique de Stephan Planck qui est l'imprimeur-libraire le plus présent dans le recueil. Cependant, comment interpréter la présence de quatre éditions imprimées dans des villes italiennes du nord de l'Italie, Pavie, Parme, Milan et Turin? La première possibilité est qu'on les ait également acquises à Rome puis ajoutées aux pièces plus anciennes a posteriori; ou bien, elles ont pu être achetées dans ces villes du nord de l'Italie pendant la retraite de l'armée française en 1495, si l'on maintient l'hypothèse. Ce serait surtout vrai de l'édition du sermo de Chieregato parue à Parme après le 12 avril 1495 ( $\mathrm{n}^{\mathrm{0}} 20$ ), dans le cas où cet opuscule faisait déjà partie de l'ensemble - nous verrons en effet qu'il pourrait s'agir d'un ajout réalisé 
au XVI ${ }^{e}$ siècle. Ajoutons que ce discours lui-même évoque la campagne de Charles VIII en faisant référence au traité signé entre Alexandre VI, Venise, Milan et le roi d'Espagne pour contrecarrer l'expédition militaire française. Bien que publiée à Turin, l'Oratio ad alexandrum VI de Petrus Cara $\left(\mathrm{n}^{\circ} 17\right)$, pour sa part, a pu être achetée à Lyon puisque les deux imprimeurs Nicolas Benedict et Giacomo Suigo ont exercé en association dans cette ville de 1496 à 1516 environ.

$\mathrm{Au} \mathrm{XVI} \mathrm{e}^{\mathrm{e}}$ siècle, le recueil factice s'est enrichi de textes plus récents. Ces éditions viennent majoritairement d'Italie mais on compte aussi parmi elles trois éditions lyonnaises, une de Vincent de Portonariis et deux de Sébastien Gryphe. Comme on peut l'imaginer, contrairement aux imprimeurs romains, publier des discours liés à la cour pontificale n'est pas une habitude pour les deux lyonnais. D'après la bibliographie lyonnaise de Sybille von Gültlingen, le discours de Guillaume Briçonnet est l'un des premiers textes publiés par Vincent de Portonariis et il apparait comme une exception ${ }^{41}$. Mis à part quelques éditions relatives à la religion, dont l'Apparatus in quinque libros decretalium d'Innocent IV ${ }^{42}$, aucun discours sacré ne semble être sorti de son atelier. Il en est de même pour Sébastien Gryphe ${ }^{43}$. Cependant, il se pourrait que la Gratulatoria oratiuncula ad Clementem VII d'Antoine Du Saix ne soit pas arrivée par hasard sous les presses gryphiennes. Certes, les deux précédentes œuvres de Du Saix, Lesperon de discipline [... $]^{44}$ (1532) et l'Oraison funebre faict et prononcée aux obseques et enterrement [...] de tres

41. Voir Henri Baudrier, op. cit., tome V, Paris, F. de Nobele, 1964, p. 404 et Sybille von Gültlingen, op. cit., tome II, 1993 (Bibiotheca bibliographica Aureliana, 141), p. 161.

42. Apparatus mirificus orbe toto celebrandus grauis simi viri domini Innocentii pape [...], Lyon, Vincent de Portonariis, 1514. Voir Sybille von Gültlingen, op. cit., tome I, 1992 (Bibiotheca bibliographica Aureliana, 135), p. 229-230.

43. Henri Baudrier, op. cit., tome VIII, 1964, p. 11-286 et Sybille von Gültlingen, op. cit., tome V, 1993 (Bibiotheca bibliographica Aureliana, 160), p. 5-216.

44. Lesperon de discipline pour inciter les humains aux bonnes lettres, stimuler a doctrine, animer a science, inuiter a touttes bonnes oeuures vertueuses et moralles par consequent pour les faire coheritiers de Jesuschrist expressement les nobles et genereux lourdement forge \& rudement lime par Noble homme Antoine du Saix Commendeur des saincts Antoine de Bourg en Bresse, 1532, [s.l.n.]. Voir Sybille von Gültlingen, op. cit., p. 41-42. 
illustre princesse Marguerite d'Autriche ${ }^{45}$ (ca. 1531-1532), longtemps attribuées aux presses de Sébastien Gryphe, sont en fait l'œuvre de Claude Nourry ${ }^{46}$. Cependant le diplomate et l'imprimeur ont certainement eu l'occasion de se fréquenter en côtoyant le sodalitium humaniste décrit par Richard Cooper ${ }^{47}$. Benoît Lecourt lui-même a connu certains de ses membres dans les années 1530. Le poète Jean Visagier, par exemple, que R. Cooper situe également parmi les habitués du sodalitium, le cite parmi ses amis dans une épître adressée à Jean de Pins, évêque de Rieux, datée du 15 mars 1537 et publiée dans le volume d'épigrammes du poète la même année chez Michel Parmentier ${ }^{48}$. Rappelons également que c'est à Sébastien Gryphe que Benoît Lecourt confie la publication de son premier ouvrage en $1533^{49}$. Aussi ces auteurs et érudits ont-ils pour le moins pu se rencontrer dans les cercles qui se formaient autour des presses de l'enseigne au griffon ${ }^{50}$. S. Gryphe publie ensuite le dernier opuscule et le plus long (47 pages) du recueil de Benoît Lecourt, les Binae orationes de Bartholomaeus Frexius ${ }^{51}$. À partir d'un noyau, que nous supposons initial, la collection a donc été étendue selon le principe de départ, avec l'ajout en ordre chronologique de huit pièces concernant les cinq papes les plus récents jusqu'au pape régnant, Paul III.

45. Oraison funebre faict et prononcee aux obsecques et enterrement du corps inhume a Brou de tresillustre princesse Marguerite Dautriche par noble homme fraire Antoine du Saix Commendeur de sainct Antoine de Bourg \& aulmosnier de Charles le tresexellent mon seigneur le duc de Sauoye [...], [s.l.n.a. vers 1532]. Voir Sybille von Gültlingen, op. cit., p. 42.

46. William Kemp, «Lesperon de discipline d'Antoine Du Saix (1532) et l'imprimeur de Pantagruel, Claude Nourry dit "Le Prince" ", Études rabelaisiennes, Tome XXXIX, Genève, Droz, 2000, p. 23-27.

47. Richard Cooper, "L’Antiquaire Guillaume Du Choul et son cercle lyonnais », dans Gérard Defaux (dir.), Lyon et l'Illustration de la langue française à la Renaissance, Lyon, ENS Éditions, 2003, p. 261.

48. Ioannis Vulteii Remensis Epigrammatum libri IIII. Eiusdem Xenia, Lyon, Michel Parmentier, 1537. Voir Henri Baudrier, op. cit., tome X, 1964, p. 399 et Sybille von Gültlingen, op. cit., tome VII, 2001 (Bibiotheca bibliographica Aureliana, 183), p. 200.

49. Aresta amorum cum erudita Benedicti Curtii Symphoriani explanation, Lyon, Sébastien Gryphe, 1533. Voir Henri Baudrier, op. cit., tome VIII, Paris, F. de Nobele, 1964, p. 68 et Sybille von Gültlingen, op. cit., tome V, 1997 (Bibiotheca bibliographica Aureliana, 160), p. 48.

50. Brigitte Biot, Barthélemy Aneau, régent de la Renaissance lyonnaise, Paris, Honoré Champion, 1996, p. 69-71.

51. Bartholomaeus Frexius, Binae orationes. Altera pro pace, per Paulum III Pont. Max. Christianis reddita, ad Christianae reipublicae heroas. Altera pro humanarum atrium studiis: habita Senis, in Comitio ad populum, Lyon, Sébastien Gryphe, 1539. Cf. annexe 1. 
Une étude matérielle plus précise du volume permet également de supposer une constitution du recueil en plusieurs étapes. Pour commencer, observons de plus près les lettres manuscrites qui numérotent les pièces du recueil. La suite des lettres de l'alphabet qui est reproduite au coin inférieur droit de la première page de presque chaque opuscule est quasiment complète et continue. On peut donc supposer que la plume à l'origine de ces marques manuscrites est celle d'un des derniers possesseurs des discours.

Pourtant, quelques anomalies se sont glissées dans cette suite dont certaines peuvent être expliquées. La pièce $n^{\circ} 6$, l'Oratio de obedientia ad Innocentium VIII de Vasco Fernandes, n’a pas été incluse dans la «numérotation » parce qu'elle était considérée comme faisant partie d'une seule édition avec le discours de Guilelmus Caorsin ( $\left.{ }^{\circ} 5\right)$ lettré $e$. Ensuite, la pièce $\mathrm{n}^{\circ} 10$, l'Oratio ad Innocentium VIII de Robertus Guiba, lettrée $s$ était vraisemblablement initialement classée après l'opuscule ${ }^{\circ} 19$, lettré $r$, avant d'être déplacée en dixième position du recueil pour que la succession chronologique des papes soit respectée. Quant au deuxième discours de Leonello Chieregato et au texte de Jason de Mayno (respectivement discours $n^{\text {os }} 20$ et 25) qui ne sont pas compris dans la cotation, ils ont pu être oubliés (de même que les pièces n ${ }^{\text {os }} 26$ et 31 ), ou plus probablement ils ont pu être ajoutés, dans une étape de constitution du recueil postérieure à celle de la numérotation. Enfin, le fait que Benoît Lecourt et son frère aient apposé leurs signatures sur les vingt-sixième et vingt-huitième opuscules, à des endroits qui semblent complétement aléatoires lorsqu'on considère le volume dans son ensemble, laisse penser que les deux frères possédaient ces discours séparément des autres avant de constituer le recueil.

Ainsi les données de publication des orationes, dates et lieux, supposent une acquisition des opuscules en deux étapes au moins. À cela s'ajoutent les informations apportées par les données matérielles du volume. Les textes qui comportent les signatures de Benoît Lecourt et de son frère ont vécu séparément des autres pièces dans leur bibliothèque. Puis les trentetrois discours ont été mis en collection et les lettres manuscrites ont été 
inscrites au bas des premières pages des discours alors réunis, peut-être pour faciliter la tâche du relieur. C'est à ce moment qu'il est possible de faire intervenir Benoît Lecourt dans la constitution du recueil. En effet, le juriste a laissé sa signature sur le discours no 28 qui est également lettré $c c$. La signature étant antérieure à la cotation, on peut alors également attribuer cette dernière à Benoît Lecourt. Ainsi, que ce soit par sa famille ou grâce au commerce du livre, il a mis la main sur un ensemble de livrets in-quarto imprimés en Italie. À cet ensemble, il a ajouté les deux pièces qu'il possédait déjà et qui portent sa signature; il a ensuite pris l'initiative d'enrichir le recueil avec des éditions plus récentes qui modifient légèrement la cohésion du volume en y associant des pièces d'origine lyonnaise, puis il a réorganisé l'ensemble. Il a déplacé le discours $\mathrm{n}^{\circ} 10$, lettré $s$, pour respecter plus scrupuleusement l'ordre chronologique des règnes des papes. Les opuscules $n^{o s}$ vingt, vingt-cinq, vingt-six et trenteet-un quant à eux ne portent pas de cote manuscrite. Ils ont donc pu être ajoutés lors de cette étape. Ils ont aussi pu être oubliés lors de l'inscription des lettres manuscrites. Ou encore, s'ils étaient bien intégrés dans la cotation d'origine, leurs lettres manuscrites ont pu être rognées par le relieur qui confectionna la reliure aux armes. D'autant que cela fut le sort d'autres lettres comme celles du discours no 29 , lettré $d d$, et du n ${ }^{\circ} 30$, lettré ee, qui ont été partiellement rognées. Enfin, Benoît Lecourt a fait réaliser une reliure arborant ses armes pour sceller l'ensemble.

\section{Épilogue}

La constitution d'une collection de livres en général et de discours imprimés en particulier est chose courante à la Renaissance. Les éditions des discours officiels constituent une production d'une importance certaine. Pour le $\mathrm{Xv}^{\mathrm{e}}$ siècle romain par exemple, elle représente environ 13,5\% de la production de textes littéraires, qui elle-même constitue $41 \%$ de la production totale ${ }^{52}$. De plus, un même discours était souvent publié

52. D’après les chiffres donnés par P. Casciano, G. Castoldi, M. P. Critelli; et al., op. cit., p. 226. 
par plusieurs imprimeurs-libraires romains ${ }^{53}$ ou encore il pouvait être réédité dans d'autres villes en Italie ou en Europe ${ }^{54}$. De toute évidence, ces éditions ont rencontré très tôt l'intérêt du public et la constitution de collections de discours imprimés est une pratique qui apparaît dès le $\mathrm{XV}^{\mathrm{e}}$ siècle. Dans un article de 2001, Denise Hillard ${ }^{55}$ décrit en effet un volume, aujourd'hui démembré, constitué par Virgilius Bovius à Rome et adressé à son frère Jacobus le 8 novembre 1489. Ces textes étaient appréciés pour les pièces d'actualité qu'ils représentaient. Les amateurs des belles-lettres trouvaient aussi en eux des modèles d'éloquence humaniste et d'application de l'art oratoire ${ }^{56}$. Des modèles qui ont peut-être été utiles à notre juriste et docteur en droit. Les discours qui composent le recueil de la British Library ont pu également servir de modèle à Benoît Lecourt pour rédiger ses propres discours ou sermons, s'il eut à en prononcer pour honorer sa charge de curé de Coise. De même que Virgilius Bovius qui termine la dédicace en tête de son recueil par ces mots: « nec temporis nostri hystoriam aliunde requires ${ }^{57}$ ", l'intérêt pour l'histoire et l'actualité politique et religieuse a pu également motiver Benoît Lecourt à constituer ce recueil. Peut-être avait-il trouvé dans ces textes matière à satisfaire un intérêt pour l'histoire, et notamment pour l'histoire de France avec cette contribution française dont nous parlions plus haut. Cet intérêt serait d'autant plus certain si Benoît Lecourt était l'auteur de

53. C'est par exemple le cas de l'Oratio in die Omnium Sanctorum habita ad Sixtum IV de Thomas de Capitaneis (discours $n^{\circ} 3$ de l'annexe 1) qui est publiée à Rome par Stephan Planck mais aussi par Eucario Silber et Bartholomaeus Guldinbeck. L'Oratio ad Innocentium papam VIII de Guilelmus Caorsin $\left(n^{\circ} 5\right)$ se retrouve elle aussi chez ces trois imprimeurs vraisemblablement au même moment. Le discours de Nicolas d'Este quant à lui $\left(\mathrm{n}^{\circ} 15\right)$, en plus de l'édition qui est sortie des presses de Stephan Planck, se vendait chez Andreas Freitag.

54. En plus de l'édition parmesane de l'Oratio ad Alexandrum VI de Giovanni Lucido Cataneo $\left(\mathrm{n}^{\circ} 21\right)$ datant du $1^{\text {er }}$ mars 1493, pouvait s'acheter à Rome une édition chez Stephan Planck et Andreas Freitag imprimée après le 5 novembre 1492. De même, notre volume contient l'édition de Pavie de l'Oratio apud Alexandrum VI de Jason de Mayno ( $\mathrm{n}^{\circ}$ 25) datant probablement du 12 janvier 1493. Tandis qu'après le 13 décembre 1492 deux éditions romaines étaient disponibles, chez Stephan Planck et Andreas Freitag ainsi qu'une édition à Leipzig chez Gregorius Böttiger. Sur ce sujet, voir aussi l'article de Holger Nickel, op. cit., p. 156-157.

55. Denise Hillard, op. cit., p. 40 et 43.

56. Ibid., p. 40.

57. Ibid., p. 40 note 4 . 
la phrase en espagnol qui encadre les armes de France sur la page de titre de l'oratio de Guillaume Briçonnet. Peut-être trouvait-il aussi dans ces textes des témoignages de la campagne d'Italie menée par Charles VIII qui avait marqué les esprits du temps notamment grâce aux relations faites par les chroniqueurs et les poètes ${ }^{58}$. Qui plus est, le volume de la British Library semble prendre naturellement sa place parmi les livres de sa bibliothèque. Ceux-ci témoignent en effet de l'intérêt de leur propriétaire pour la papauté et son histoire. Le recueil de la British Library est par exemple à mettre en parallèle avec deux autres ouvrages ayant appartenu au juriste: les Vitae pontificum de Platina dont les marges sont parcourues d'annotations de Benoît Lecourt et de Léonard ainsi que le catalogue manuscrit des prophéties des papes écrit de la main du juriste ${ }^{59}$.

Grâce aux diverses traces laissées par les possesseurs et à partir d'éléments bibliographiques et matériels, nous pouvons donc aujourd'hui tenter de reconstituer les destinées des recueils factices qui se sont constitués au cours des siècles. Tenter de déchiffrer ces volumes, faisceaux de données, condensés d'histoire et de pratiques culturelles, à la fois témoins et tributaires du temps qui passe, nous permet de mieux comprendre les conditions de transmission des textes imprimés, les pratiques bibliophiliques et les centres d'intérêts des gens du temps. Nous avons pu ici approcher Benoît Lecourt et sa bibliothèque et il nous semble déjà les connaître davantage.

58. Ivan Cloulas, «La découverte de l'Italie par Charles VIII », dans Jean Balsamo (dir.), Passer les Monts. Français en Italie - l'Italie en France (1494-1525), Paris, Honoré Champion, 1998, p. 127 138. Voir aussi Jean-Pierre Seguin, « L'information à la fin du $\mathrm{XV}^{\mathrm{e}}$ siècle en France, pièces d'actualité imprimées sous le règne de Charles VIII », Arts et Traditions populaires, $4^{\mathrm{e}}, \mathrm{n}^{\circ} 4$, octobre-décembre 1956, p. 309-330.

59. Ces volumes sont conservés à la Bibliothèque municipale de Lyon (Rés Ms 195, T. 1 et T. 2). 


\section{Annexe 1 Liste des éditions ${ }^{60}$}

Les chiffres entre crochets de la première colonne correspondent au classement des discours dans le volume. Les lettres reproduisent la cotation manuscrite d'origine. La présence d'un tiret à la place d'une lettre indique que l'opuscule ne comporte pas de lettre manuscrite.

[1]a. Pius II (Aeneas Sylvius Piccolomini)

Oratio coram Calixto III de obedientia Friderici III

[Rome, S. Planck, 1488-90]

In- $4^{\circ}$, [5-1 bl.] f., goth.

ISTC (ip00731000); BMC IV.95; British Library (IA.18602)

[2]b. Giustiniano, Bernardo

Oratio habita apud Sixtum IV contra Turcos

[Rome, S. Planck, 1481-87]

In- $4^{\circ}$, [7-1 bl.] f., goth.

ISTC (ij00610000); BMC IV.90; British Library (IA.18629)

[3]c. Capitaneis, Thomas ex

Oratio in die Omnium Sanctorum habita ad Sixtum IV

[Rome, S Planck, après le $1^{\mathrm{er}}$ Nov. 1483]

In- $4^{\circ},[6]$ f., goth.

ISCT (ic00122000); BMC IV.82; British Library (IA.18343)

60. Abréviations utilisées: ISTC: Incunabula Short Title Catalogue [en ligne], Londres, British Library, [consulté le 04/12/2013], disponible à l'adresse: <http://www.bl.uk/catalogues/istc/> ; USTC: Universal Short Title Catalogue [en ligne], Londres, British Library, [consulté le 04/12/2013], disponible à l'adresse <http://ustc.ac.uk/index.php>; Baudrier: Henri Baudrier, Bibliographie Lyonnaise. Recherches sur les imprimeurs, libraires, relieurs et fondeurs de lettres de Lyon au XVI ${ }^{e}$ siècle, tome V, VII et VIII, Paris, F. de Nobele, 1964; Begey 1961: Marina Bersano Begey, Le cinquecentine pièmontesi, tome I, Turin, Tipographia Torinese, 1961, 551 p.; BMC: Catalogue of books printed in the xvth century now in the British Museum. Part IV. Italy: Subiaco and Rome, London, British Museum, 1916, XVI-145 p. 
[4]d Geraldinus, Antonius

Oratio in obsequio nomine Ferdinandi et Elisabeth Innocentio VIII exhibio, 19 sept. 1486

[Rome, S. Planck, 1488-91]

In- $4^{\circ}$, [6] f., goth.

ISTC (ig00161000); BMC IV.93; British Library (IA.18445)

[5]e. Caorsin, Guilelmus

Ad Innocentium papam VIII oratio

[Rome, S. Planck, après le 28 janv. 1485]

In- $4^{\circ}$, [2] f., goth.

ISTC (ic00107000); BMC IV.84; British Library (IA.18399)

[6]-. Fernandes, Vasco

Oratio de obedientia ad Innocentium VIII

[Rome, S. Planck, 1488-90]

In- $4^{\text {o, }}$ [6] f., goth.

ISTC (if00097900); BMC IV.93; British Library (IA.18367)

[7]f. Cadratus, Petrus

Oratio ad Innocentium VIII habita, anno 1485, 11 februarij.

Publié avec:

Andreoccius de Shinucciis (Ghinuciis)

Oratio pro Senensibus ad Innocentium VIII

[Rome, S. Planck, après le 11 Fév. 1485]

In- $4^{\circ}$, [4] f., goth.

ISTC (ic00015000); BMC IV.85 (un autre exemplaire); British

Library (IA.18407)

[8]g. Dalberg, Johannes de

Gratulatio Innocentio VIII dicta

[Rome, S. Planck, après le 6 juil. 1485]

In- $4^{\circ}$, [4] f., goth.

ISTC (id00001000); BMC IV.86; British Library (IA.18428) 
[9]h. Marlianus, Johannes Franciscus

Oratio habita apud Innocentium VIII

[Rome, S. Planck, après le 29 juin 1485]

In- $4^{\circ}$, [4] f., goth.

ISTC (im00275000); BMC IV.85; British Library (IA.18423)

[10]s. Guiba, Robertus

Oratio ad Innocentium VIII in obendientia praestanda

In- $4^{\circ}$, [1] f., goth.

[Rome, S. Planck, après le 10 juin 1485]

ISTC (ig00550000); BMC IV .85; British Library (IA.18371)

[11]i. Chieregato, Leonello

Oratio in funere Innocentii VIII

[Rome, E. Silber, après le 28 juil. 1492]

In- $4^{\circ}$, [4] f., rom.

ISTC (ic00453000); BMC IV.113; British Library (IA.18937)

[12]k. Carvajal, Bernardino de

Oratio de eligendo summo pontifice habita ad sacratissimum senatum cardinalium Innocentio octavo de mortuo

[Rome, E. Silber, après le 6 août 1492]

In $-4^{\circ},[10]$ f., rom.

ISTC (ic00224000); BMC IV.113; British Library (IA.18940)

[13]l. Montanus, Marcus

Oratio pro Rhodiorum obedentia ad Alexandrum VI

[Rome, E. Silber, après le 10 mars 1493]

In $-4^{\circ}$, [4] f., rom.

ISTC (im00824000); BMC IV.115; British Library (IA.18977)

[14]m. Becchi, Gentile, Évêque d'Arezzo

Oratio Florentinorum corem Alexandro VI habita

[Rome, E. Silber, après le 28 Nov. 1492] 
In- $4^{\circ}$, [5 - 1 bl.] f., rom.

ISCT (ib00291600); BMC IV.114; British Library (IA.18965)

[15]n. Este, Nicolaus Maria de

Oratio pro Generoso Hercule Estensi Ferrariae Duce secondo habita ad Alexandrum VI

In- $4^{\circ}$, [4] f., rom.

Rome, S. Planck, 9 Janv. 1493

ISTC (ie00110000); BMC IV.97; British Library (IA.18543)

[16]o. Floridus, Bartholomaeus

Oratio confoederationis initiae inter Alexandrum VI et Venetorum, Mediolani et Bari duces

[Rome, E. Silber, après le 25 avril 1493]

In $-4^{\circ}$, [4] f., rom.

ISTC (if00228200); BMC IV.115; British Library (IA.18991)

[17]p. Cara, Pietro

Oratio ad Alexandrum VI

[Turin, Nicolaus de Benedictus et Jacobus Suigus, 1494]

In $-4^{\circ}$, sig. $a^{6} b^{4}$, [10] f., rom.

ISTC (ic00130200); BMC VII.1058; British Library (IA.32445)

[18]q. Spinola, Giacomo

Oratio gratulatoria ad Alexandrum VI nomine Genuensium habita

[Rome, S. Planck, après le 12 Déc. 1492]

In $-4^{\circ}$, sig. $a^{6},[6]$ f., rom.

ISTC (is00683000); BMC IV.96; British Library (IA.18519)

[19]r. Manilio, Jean Antoine

Oratio pro Britonoriensibus ad Alexandrum VI

[Rome, S. Planck, août 1492 ?]

In $-4^{\circ}$, [2] f. (imp.), rom.

ISTC (is00683000); BMC IV.96; British Library (IA.18519) 
[20]-. Chieregato, Leonello

Sermo in publicatione confoederationis inter Alexandrum VI

et Romanorum et Hispaniae reges, Venetorumque ac

Mediolanensium duces

Publié avec: Alexandre VI,

Bulla Quoniam pro communi, 8 avril 1495

[Milan, Ph. De Mantegatiis, après le 12 avril 1495]

In $-4^{\circ}$, sig. $a^{4}$, [4] f., goth.

ISTC (im00200000); BMC VI.787; British Library (IA.18531)

[21]t. Cataneo, Giovanni Lucido

Oratio ad Alexandrum VI

Parma, A. Ugoletus, $1^{\text {er }}$ mars 1493

In- $4^{\circ}$, sig. $\mathrm{A}^{10}$, [9 - 1 bl.] f., rom.

ISTC (ic00277500); BMC VII.945; British Library (IA.30348a)

[22]u. Badoaro, Sebastiano

Oratio ad Alexandrum VI in praestanda

Venetorum obedientia

[Rome, S. Planck, après le 17 Déc. 1492]

In- $4^{\circ}$, [2] f., rom.

ISTC (ib00007000); BMC IV.97; British Library (IA.18525)

[23]x. Tegrini, Niccolò

Oratio per obedientia praestanda Alexandro VI

[Rome, S. Planck, après le 26 Oct.1492]

In $-4^{\circ},[4 \mathrm{f}]$., rom.

ISTC (it00565000); BMC IV.96; British Library (IA.18510)

[24]y. Senensium obedientia publica [ad Alexandrum VI]

[Rome, A. Freitag, après le 15 Oct. 1492]

In $-4^{\circ}$, [2] f., goth.

ISTC (is00440000); BMC IV.136; British Library (IA.19332) 
[25]-. Mayno, Jason de

Ad Illustrissimum et sapientissimum Principes Ludouicus

Sfortiam Vicecomites barri ducem

Publié avec:

Oratio apud Alexandrum VI habita pro Mediolanensium

principe

[Pavia, Antonius de Carcano, 12 Janv.? 1493]

In- $4^{\circ}$, [10] f., goth., titres rubriqués

ISTC (im00410000); BMC VII.999; British Library (IA.31363)

[26]-. Briçonnet, Guillaume

Coram Iulio II pro francorum Rege Ludovico XII. appologia

Lyon, V. de Portonariis, 12 octobre 1507

In $-4^{\circ}$, sig. a- $e^{4},[20]$ f., rom.

Baudrier V. 404; British Library (IA.18602.(27.))

[27]bb. Nivarius, Eustachius

Oratio habita Romae coram Iulio II

Bologne, B. Hectores, 13 juillet 1506

In $-4^{\circ}$, sig. $A^{8}$, [8] f., rom.

British Library (IA.18602.(28))

[28]cc. Possedarski, Stephan

Oratio habita apud Leonem X pro domino Ioanne Torquato

Comite Corbavie Defensore Crovacie

[Rome, Stefano Guillery, 1516]

In- $4^{\circ}$, sig. $A^{4}$, [4] f., rom.

USTC: 851056; British Library (IA.18602.(29.))

[29]dd. Lasco, Joannes de

Oratio ad Leonem X, in Obedientia Sigismundi Regis

Poloniae Magniducis Lituaniae Russiae Prussiaeque habita anno M.D.XIII.

[Rome, M. Silber, 1513] 
In $-4^{\circ}$, sig. $A^{4}$, [4] f., rom.

USTC: 837422; British Library (IA.18602.(30.))

[30]ee. Parpalia, Bernardinus

Ad Leonem $X$ oratio

Turin, J. Angelus \& B. de Silva, [1514-20]

In- $4^{\circ}, 4 \mathrm{f}$., rom.

Begey 1961, I, no 408; British Library (IA.18602.(31.))

[31]- Flaminius, Joannes Antonius

Ad Adrianum VI epistola

Bologne, H. de Benedictis, 1523

In- $4^{\circ}$, sig. A-B ${ }^{4} C^{6}$, [14] f., rom.

British Library (IA. 18602.(32.))

[32]gg. Du Saix, Antoine

Gratulatoria oratiuncula ad Clementem VII super faustissimo ipsius in Galliam adventu

Lyon, S. Gryphe, 1534

In- $4^{\circ}$, sig. $a-b^{4}$, [8] f., rom.

Baudrier VIII. 76-77; British Library (IA.18602.(33.))

[33]hh. Frexius, Bartholomaeus

Binae orationes. Altera pro pace, per Paulum III Pont. Max.

Christianis reddita, ad Christianae reipublicae heroas.

Altera pro humanarum atrium studiis:habita Senis, in Comitio ad populum.

Lyon: S. Gryphe, 1539

In $-4^{\circ}$, sig. a-f $\mathrm{f}^{4}, 47$ [1] p., rom. et ital.

Baudrier VIII. 123; British Library (IA.18602.(34.)) 


\section{Annexe 2}

Chronologie des papes, classement des discours dans le recueil et nombre de discours pour chaque souverain pontife.

\begin{tabular}{|c|c|c|c|}
\hline Pape & Règne & Discours & $\begin{array}{c}\text { Nombre } \\
\text { total } \\
\text { de discours } \\
\text { par pape }\end{array}$ \\
\hline Calixte III & 8 avril $1455-6$ août 1458 & $\mathrm{~N}^{\circ} 1$ & 1 \\
\hline Pie II & 27 août $1458-14$ août 1464 & l & 0 \\
\hline Paul II & 30 août $1464-26$ juillet 1471 & I & 0 \\
\hline Sixte IV & 9 août $1471-12$ août 1484 & No $2-3$ & 2 \\
\hline Innocent VIII & 20 août $1484-25$ juillet 1492 & No $4-12$ & 9 \\
\hline Alexandre VI & 11 août $1492-18$ août 1503 & $\mathrm{~N}^{\circ} 13-25$ & 13 \\
\hline Pie III & 22 septembre 1503 - 18 octobre 1503 & I & 0 \\
\hline Jules II & $1^{\text {er }}$ novembre $1503-21$ février 1513 & No $26-27$ & 2 \\
\hline Léon X & 9 mars $1513-1^{\text {er }}$ décembre 1521 & $\mathrm{~N}^{\circ} 28-30$ & 3 \\
\hline Adrien VI & 9 janvier 1522 - 14 septembre 1523 & $\mathrm{~N}^{\circ} 31$ & 1 \\
\hline Clément VII & 19 novembre 1523 - 25 septembre 1534 & $\mathrm{~N}^{\circ} 32$ & 1 \\
\hline Paul III & 13 octobre $1534-10$ novembre 1549 & $\mathrm{~N}^{\circ} 33$ & 1 \\
\hline
\end{tabular}




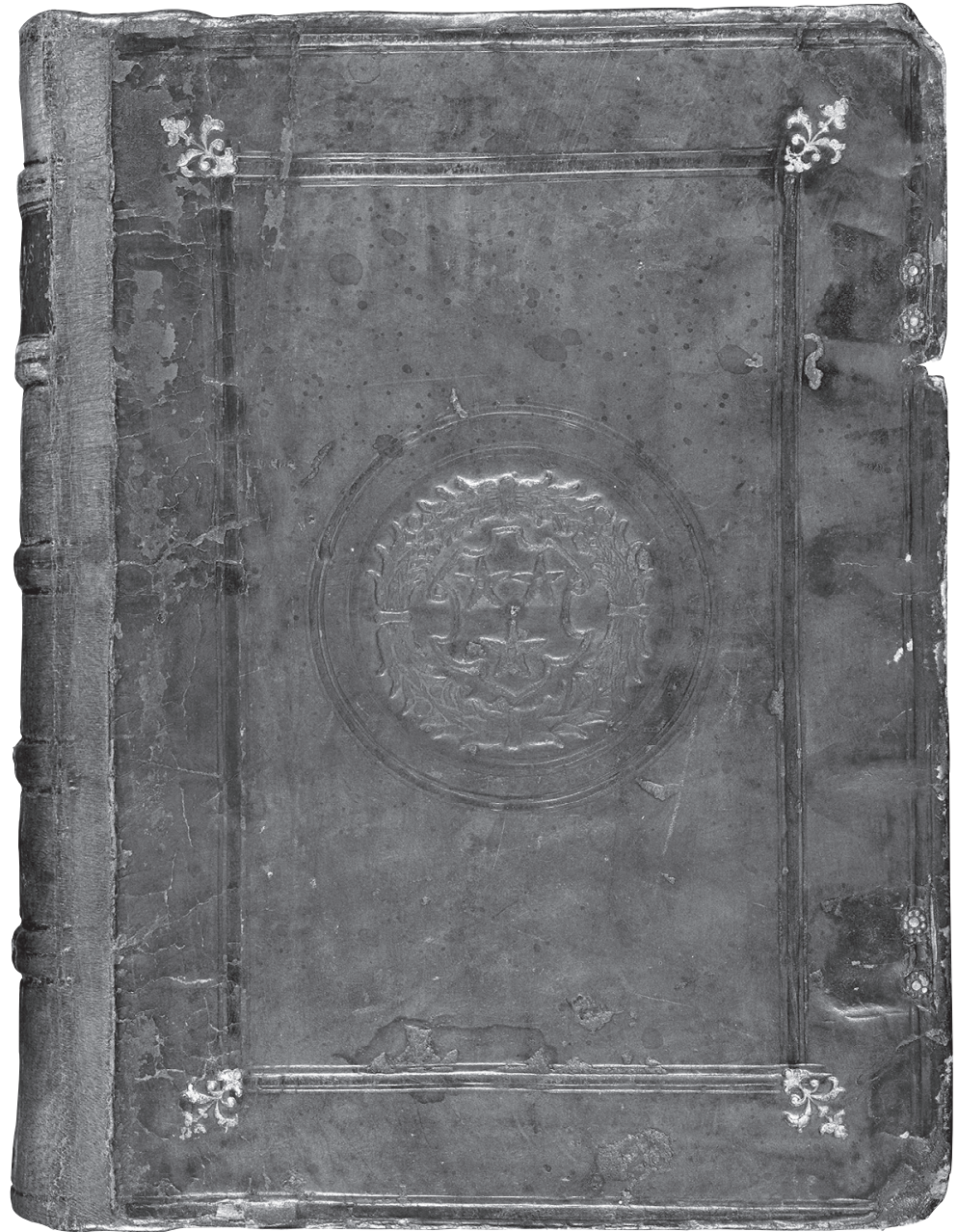

Figure 1

Reliure aux armes de Benoît Lecourt - Plat supérieur. 


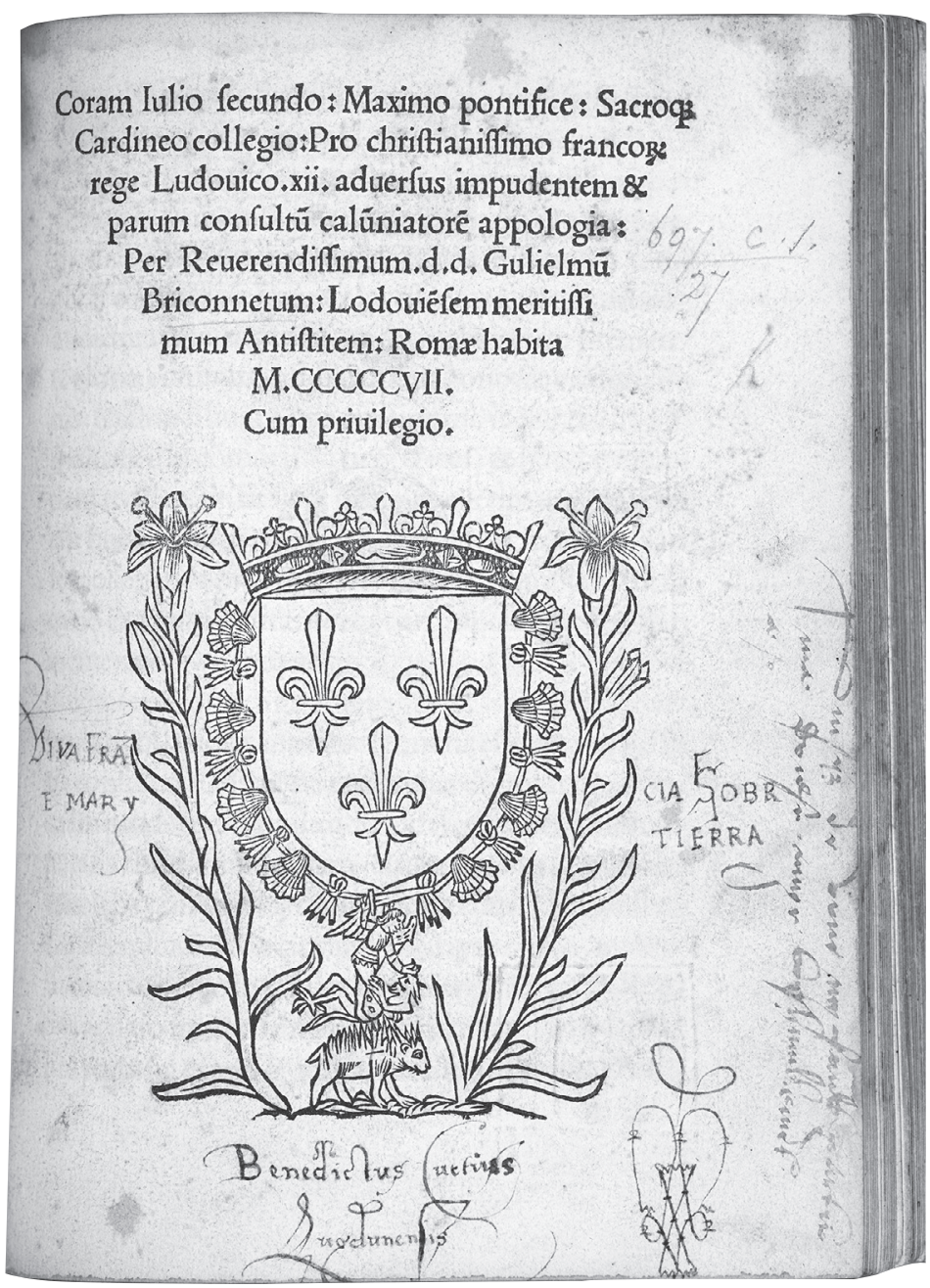

Figure 2

Guillaume Briçonnet, Coram Iulio II pro francorum Rege Ludovico XII appologia, Lyon, V. de Portonariis, 12 octobre 1507;

In-4 ${ }^{\circ}$, sig. a-e4, [20] f., rom - Page de titre. 
